\title{
The Sun shadow observation with the ANTARES neutrino telescope
}

\author{
The ANTARES ${ }^{\dagger}$ Collaboration \\ $\dagger$ http://antares.in2p3.fr/Collaboration/index2.htm \\ E-mail: tommaso.chiarusidbo.infn.it, Luigi.fuscodapc.in2p3.fr, \\ andrey.romanovege.infn.it, matteo.sanquinetiege.infn.it, \\ federice.versariabo.infn.it
}

The ANTARES detector is an abyssal undersea neutrino telescope in the Mediterranean Sea. The search for point-like neutrino sources is one of the main goals of ANTARES, so a reliable way to evaluate the detector angular resolution and pointing accuracy is needed. In a previous analysis, the deficit in the atmospheric muon flux in the direction of the Moon induced by absorption of cosmic rays was investigated. It has allowed the estimation of the angular resolution and of the pointing accuracy of the detector. In this work the same approach was applied to measure the Sun shadow effect. This analysis is based on the ANTARES data taken in the interval between 2008 2015. According to Monte Carlo simulation the expected statistical significance of the analysis is $2.9 \sigma$.

Corresponding authors: Tommaso Chiarusi ${ }^{1}$, Luigi Antonio Fusco*2, Andrey Romanov ${ }^{3}$, Matteo Sanguineti ${ }^{4,5}$, Federico Versari ${ }^{1,6}$,

${ }^{1}$ INFN, Sezione di Bologna, Viale Berti-Pichat 6/2, 40127 Bologna, Italy

2 APC, Univ Paris Diderot, CNRS/IN2P3, CEA/Irfu, Obs de Paris, Sorbonne Paris Cité, France

3 Moscow State University, Skobeltsyn Institute of Nuclear Physics, Leninskie gory, 119991 Moscow, Russia

${ }^{4}$ INFN, Sezione di Genova, Via Dodecaneso 33, 16146 Genova, Italy

5 Dipartimento di Fisica dell'Università, Via Dodecaneso 33, 16146 Genova, Italy

${ }^{6}$ Dipartimento di Fisica e Astronomia dell'Università, Viale Berti Pichat 6/2, 40127 Bologna, Italy

36th International Cosmic Ray Conference -ICRC2019-

July 24th - August 1st, 2019

Madison, WI, U.S.A.

${ }^{*}$ Speaker. 


\section{Introduction}

The ANTARES undersea neutrino telescope is currently in operation for more than 10 years [四]. The main goal of the telescope is to detect neutrino point-like sources, so it is critically important to correctly estimate the angular resolution and the pointing accuracy of the telescope. One of the methods to evaluate the angular resolution is based on the detection of the so-called "Moon and Sun shadow". The shadow is the atmospheric muon flux deficit in the region around the celestial object due to its absorption of primary cosmic rays. A similar measurement has been performed by

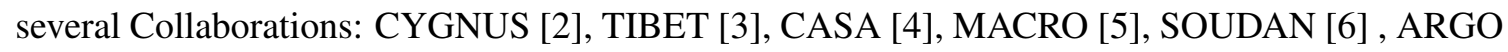
[] and also IceCube [ [8]. The measurement of the Moon shadow with ANTARES was published in [G]. In this proceeding we report the preliminary results of the Sun shadow analysis using the ANTARES 2008-2015 data sample, corresponding to a total live-time of around 2800 days. The raw data were processed through the standard ANTARES reconstruction chain [ए]], obtaining a final sample $3 \times 10^{6}$ events reconstructed as down-going muons.

\section{Optimisation of quality cuts}

A Monte Carlo (MC) simulation has been produced and exploited in order to optimize the selection criteria of the analysis. Such a simulation, based on down-going muon events generated at the detector level with the MUPAGE code [W], includes the propagation of the muons in the instrumented volume, the induced emission of Cherenkov light, the light propagation to the optical modules and the digitised response of the PMTs, i.e. the so called "hits". The simulated hits were then processed through the same mentioned reconstruction chain, as done for the real data. The generation of the MC sample is subdivided in different batches corresponding to the actual datataking periods, denominated "runs", into which the above mentioned live-time span was subdivided (run-by-run MC simulation [ए2]). The trade-off between the accuracy of the simulations and the occupancy of the computing resources, exploited to produce the MC sample, limited the MC muon statistics to $1 / 3$ of the actual expected one.

Figures of merit of the tracks are determined by means of two quality parameters: $\Lambda$, which varies linearly with the logarithm of the reconstructed track likelihood, and $\beta$, the angular error associated to the reconstructed direction [[13]. The quality cuts optimisation is based on the hypothesis test approach, similarly to what reported in [Q] . In the present case, $H_{0}$ hypothesis corresponds to the absence of the Sun, while $H_{1}$ hypothesis is compliant with the presence of the Sun. The cut optimisation is then achieved by maximising the separation between $H_{0}$ and $H_{1}$. Two different MC simulation sets are prepared. One sample includes the Sun shadow effect which is obtained by removing the muons generated within the Sun disk, assuming a radius of $0.26^{\circ}$, and the other one is without this effect. For each of the two MC samples a one dimensional histogram is built with the distribution of events as a function of the angular distance $\delta$ with respect to the Sun position, up to $10^{\circ}$. Such a histogram is subdivided into $N_{\text {bins }}=25$ bins, each one sized $\Delta \delta=0.4^{\circ}$ and corresponds to an annulus of increasing radius centred on the Sun. The content of each bin is normalised to the corresponding annulus area, resulting in an event density.

The used test statistic is defined as $\lambda=-2 \log \frac{L_{H_{1}}}{L_{H_{0}}}$, with $L_{H_{0}}$ and $L_{H_{1}}$ are the likelihoods obtained under the $H_{0}$ and $H_{1}$ hypotheses. The expression for $\lambda$ can be written as: 


$$
\lambda=2 \sum_{i=1}^{N_{b i n s}}\left[\mu_{i}-v_{i}+n_{i} \ln \frac{v_{i}}{\mu_{i}}\right]
$$

where $v_{i}$ and $\mu_{i}$ are respectively the expected bin content given $H_{0}$ and $H_{1}$ hypotheses. The two probability distributions of $\lambda, p\left(\lambda \mid H_{0}\right)$ and $p\left(\lambda \mid H_{1}\right)$, valid separately under the hypotheses $H_{0}$ and $H_{1}$, respectively, are obtained by means of pseudo-experiments (PEs). The number of events in the $i$-th bin $n_{i}$ is determined using $10^{6}$ random values generated according to a Poisson distribution with expectation values equal to $v_{i}$ and $\mu_{i}$.

In order to find the best cuts on $\Lambda$ and $\beta$, the hypothesis test procedure was repeated for different sets of cuts applied to the Monte Carlo sample, which includes the events with $\Lambda>-6.5$ and $\beta<1^{\circ}$. Fig. $⿴$ represents the distribution $p\left(\lambda \mid H_{0}\right)$ (black curve) and $p(\lambda \mid H 1)$ (red curve) for the optimised quality cuts: $\Lambda_{c u t}=-6.08$ and $\beta_{c u t}=1^{\circ}$. The optimal cut corresponds to $\beta<1^{\circ}$ which is on the border of the pre-selected data sample. The future analysis will attempt a broader events pre-selection and sensitivity may get improved. The value of the expected significance using this set of cuts is $2.9 \sigma$. Fig. $\square$ shows the dependence between the statistical significance and the cuts.

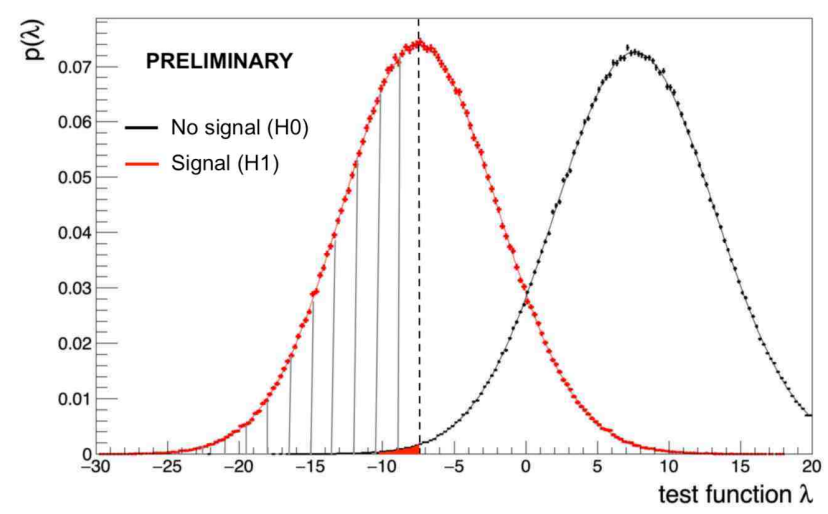

Figure 1: The test function $\lambda$ distribution for "Sun shadow" hypothesis (red curve) and "no Sun shadow" hypothesis (black curve). The dashed area corresponds to the $50 \%$ of the pseudo-experiments where $H_{1}$ hypothesis is correctly identified. The shaded area quantifies the expected median significance $(2.9 \sigma)$ to reject the no-Sun hypothesis in favour of the presence of the Sun. 


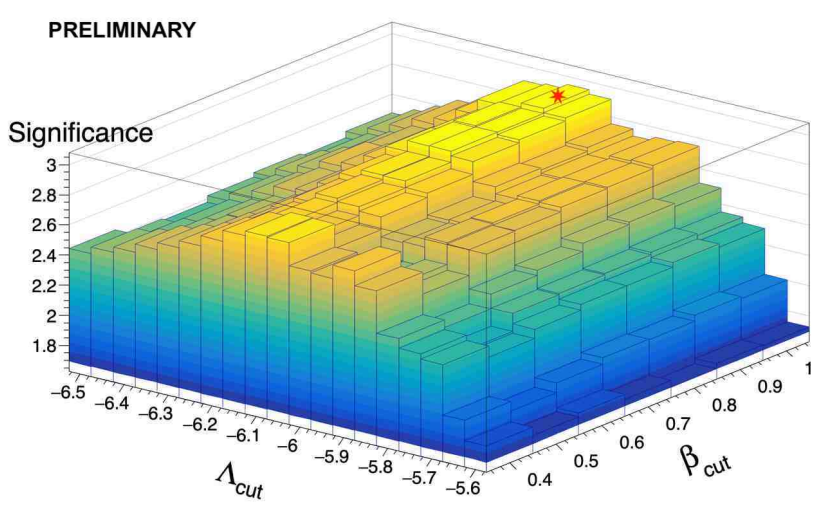

Figure 2: Statistical significance of the Sun shadow detection during the period from 2008 to 2015 based on Monte Carlo sample as a function of $\Lambda$ and $\beta$. Best cuts on $\Lambda$ and $\beta: \Lambda_{\text {cut }}=-6.08$ and $\beta_{\text {cut }}=1^{\circ}$, for a significance is $2.9 \sigma$.

\section{Sun-shadow 1D profile distribution and angular resolution estimation}

The reconstructed events from the 2008-2015 ANTARES data sample have been selected with the optimized cuts described above and the muon density close to the Sun region is derived. The events were binned using concentric rings around the Sun centre up to an angular distance of $10^{\circ}$ with bin size of $0.4^{\circ}$. The muon density versus the distance from the Sun is presented in Fig. 目. The muon deficit is clearly visible close to the nominal Sun position. For the estimation of the angular resolution, the Sun shadowing effect is assumed to follow a Gaussian distribution with standard deviation $\sigma_{\text {res }}$, which is the detector angular resolution itself [Q]. A similar approach has already been followed by $[[],[]]$. The number of expected events is evaluated by fitting the distribution in Fig. 3 with the following function [6]:

$$
\frac{d N}{d \delta^{2}}=k\left(1-\frac{R_{\text {Sun }}^{2}}{2 \sigma_{\text {res }}^{2}} e^{-\frac{\delta^{2}}{2 \sigma_{\text {res }}^{2}}}\right),
$$

where $k$ is the event density level far from the nominal Sun position, $R_{\text {sun }}$ is the Sun angular radius $\left(0.26^{\circ}\right), \delta$ is the angular distance from the Sun centre. The angular resolution resulting from the fit is $\sigma_{\text {res }}=0.99^{\circ} \pm 0.34^{\circ}$. This result is in a good agreement with the Monte Carlo expectations leading to $\sigma_{\text {res }}^{M C}=0.98^{\circ} \pm 0.33^{\circ}$ (Fig. 团). Finally, the fitted angular resolution is compatible to the Moon shadow analysis result at $1 \sigma$ level [Q]

When cosmic rays propagate from the Sun to the Earth they could be influenced by the combined effect of the Sun magnetic field, the interplanetary space and the Earth magnetic field, so it can lead to the blurring of the shadow. This hypothesis was checked by dividing the data into two samples with roughly equal statistics. The first one covers the period from 2008 to 2011, when the Sun activity was low, while the second one covers the period from 2012 to 2015, when the Sun activity was high. However, the statistics in these two samples is too low. So the conclusions about magnetic fields influence can not be obtained. 


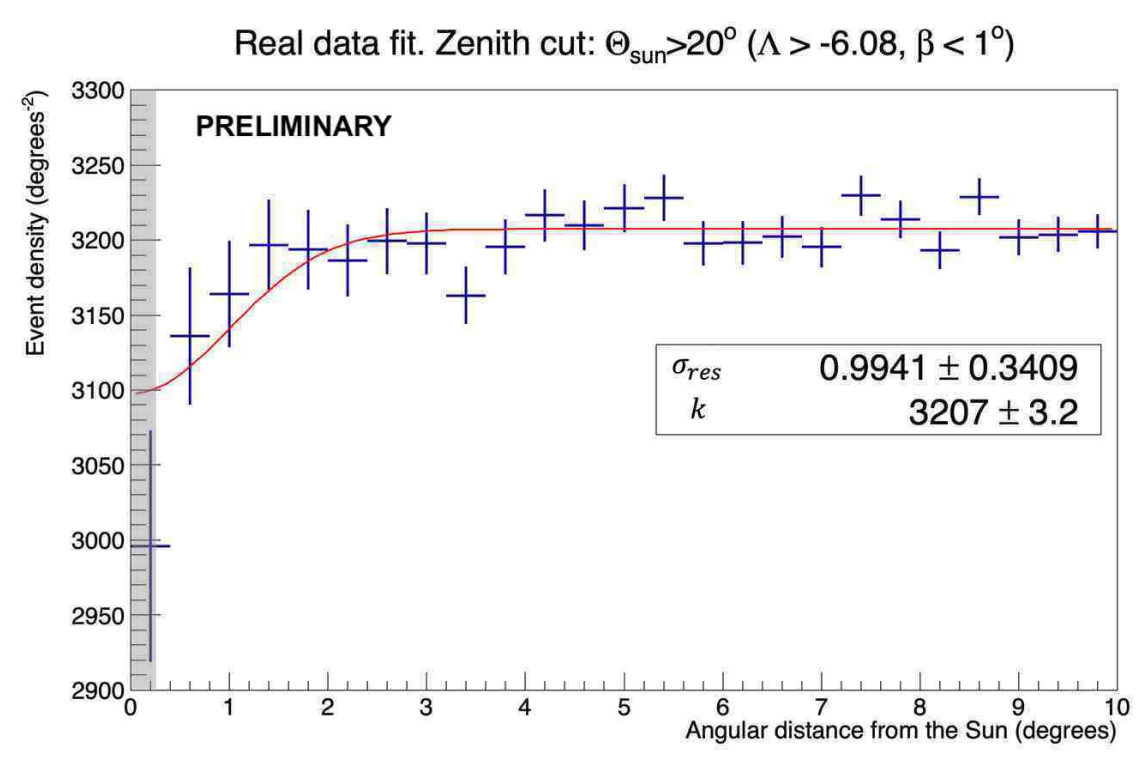

Figure 3: The muon events density as a function of the angular distance $\delta$ from the Sun centre based on the data sample taken in the period from 2008 to 2015. The muon shadowing is evident close to the nominal Sun region. The shaded area corresponds to the Sun angular radius $\left(0.26^{\circ}\right)$

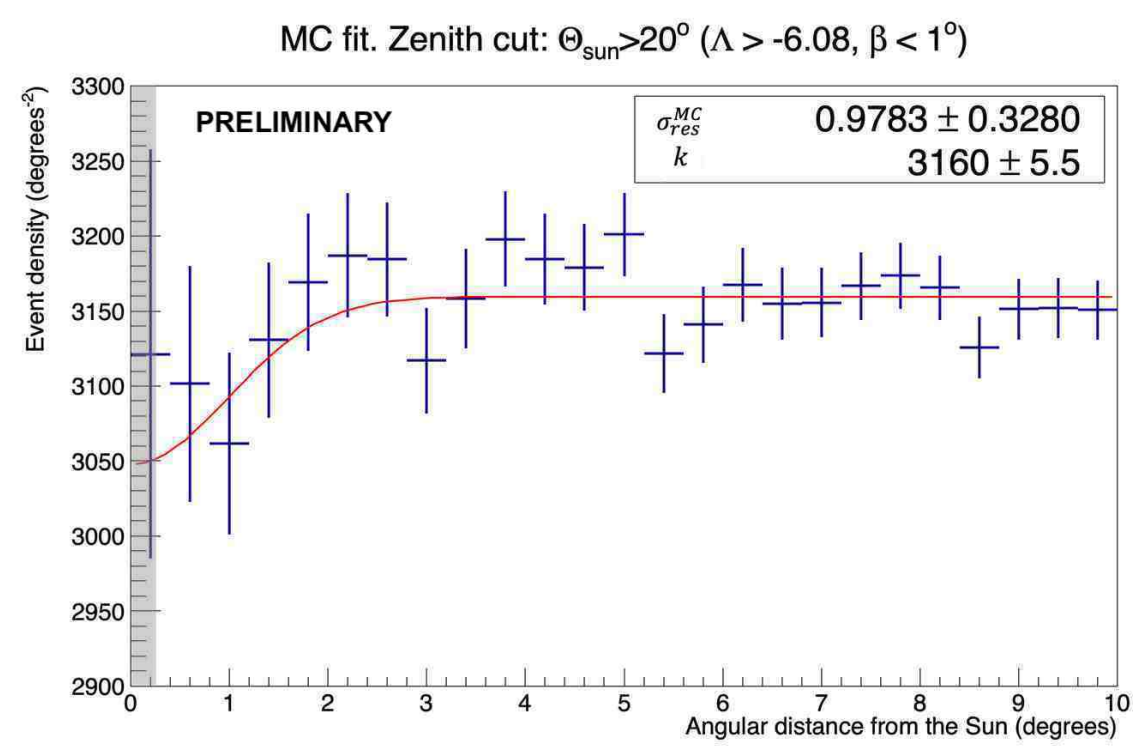

Figure 4: The muon events density for Monte Carlo sample with the generated events excluded within the Sun disk (shaded area) 


\section{Conclusions}

In this work the analysis of the Sun shadow effect was performed based on the 2008-2015 ANTARES data sample, corresponding to a total live-time of around 2800 days. The expected value of the statistical significance for the ANTARES Sun shadow analysis is $2.9 \sigma$. The data sample shows a clear deficit in the region close to the nominal Sun position and the estimated detector angular resolution for atmospheric downward-going muons is $\sigma_{\text {res }}=0.99^{\circ} \pm 0.34^{\circ}$. The angular resolution value is compatible to the Moon shadow analysis result at $1 \sigma$ level. A larger statistical significance for the Sun shadow will be possible by increasing the data sample with events from the latest reconstructed runs (up to 2017). With such an improved statistics the study of the detector pointing performance will be then more effective obtaining the $68 \%, 90 \%$ and $95 \%$ CL contours of the Sun Shadow in the field of view around the expected direction of the Sun.

\section{References}

[1] M. Ageron et al. (ANTARES Coll.), Nuclear Instruments \& Methods in Physics Research A656, $11-38$ (2011)

[2] D. E. Alexandreas et al., Phy. Rev., D43:1735-1738 (1991)

[3] M. Amenomori et al., Phys. Rev., D47:2675-2681 (1993).

[4] A. Borione et al., Phys. Rev., D49:1171-1177 (1994).

[5] M. Ambrosio et al., Phys. Rev., D59:012003 (1999).

[6] H. Cobbs et al. , Phys. Rev., D61:092002 (2000).

[7] ARGO-YBJ Collaboration, Phys. Rev., D.84:022003 (2011).

[8] ICECUBE collaboration, The Astrophysical Journal 872.2 (2019): 133.

[9] Albert, A., et al. (ANTARES Coll.) The European Physical Journal C 78.12 (2018): 1006.

[10] S. Adrian-Martinez et al. (ANTARES Collaboration), JCAP 1303, 006 (2013)

[11] G. Carminati, A. Margiotta, M. Spurio, Comput. Phys. Commun. 179, 915-923 (2008)

[12] L.A. Fusco, A. Margiotta, EPJ Web Conf. 116, 02002 (2016)

[13] A. Albert et al. Phys. Rev. D 96, 082001 (2017) 